\title{
College's Academic Organization Construction Based on Perspective of Disciplines
}

\author{
Min Zhou \\ Academy of Modern Logistic Industry \\ Beijing Wuzi University, \\ Beijing, P.R.C. \\ Lilytina74@yahoo.com
}

\begin{abstract}
The development of disciplines is consistent with the trend of modern science. It follows a trend of integration-differentiation-integration again. The overall trend is both differentiated and integrated. The disciplinary system is a dynamic structure of the condensed subject groups. With the development of disciplines, the formality of academic organization and relationship of disciplines in college have been going through constant changes. From the three aspects of academic organizational structure, operation mechanism of organization, and organizational culture, this article makes recommendations on the current construction of college academic organizations.
\end{abstract}

Keywords-modern science; discipline; college academic organization

\section{THE LAW OF DISCIPLINE DEVELOPMENT AND THE EVOLUTION OF COLLEGE ACADEMIC ORGANIZATION}

\section{The Connotations of Discipline and College Academic Organization}

$<<$ CiHai $>>$ has two terms for the subject of disciplines. 1. The academic classification, which means certain fields of science or a branch of science, such as physics and biology in natural science, and history and education in social sciences, and so on.2. Subjects of education, a basic unit in college's educational content. Researchers from educational field in China always define disciplines in three aspects: one is the branch of knowledge, the second is the subject of school education, the third is the academic organization. In 2011, the Academic Degrees Committee of the State Council and Ministry of Education issued $<<$ The subject directories of new degrees and personal training (201 ) >>, currently a total of 13 categories of discipline, 110 level-A subjects.

Academic organization refers to an organization with purpose to engage in scientific research and to promote advancement of science and technology. It is asocial entity with a high degree of autonomy that makes rational management and coordination in order to inherit knowledge and make innovations, such as various academic and research institutions, academic organizations, associations, and societies, etc. College is an academic organization that uses knowledge as material and combines units of disciplines. Discipline is the essential characteristic of college academic organization. (Burton R. Clark , 1994)College is an organization that obtains, conveys, and applies advanced knowledge. It bears responsibility for personal training, scientific study, and community service. Colleges or all organizations within a college, such as schools, departments, and scientific research base, have the fundamental elements and the common virtual of an academic organization.

\section{The Law of Discipline Development and the Evolution of College Academic Organization}

The development of discipline is consistent with the development of modern science. Before 16th century, the low level of productive forces determined that science was basically an empirical knowledge and hasn't evolved into a complete form of discipline. Natural science, literature, history and philosophy were all in one category, that is, one natural philosophy subject. Since the appearance of modern science in 16th century till early 19th century,, with the landmark separation of natural science and philosophy, natural science and social science were separated from philosophy. In the natural science disciplines, the disciplinary system of astronomy, mathematics, mechanics started to become more complete, and physics, chemistry, biology and other disciplines began to be established.

In social science disciplines, economics, literature, history, sociology, psychology, pedagogy, and so on formed a more rigorous disciplinary system. From the 19 th century to the 20 th century, modern science and technology developed rapidly and new disciplines were created continuously. Since the middle of the 20th century, systematic science started to grow. Interdisciplinary and multidisciplinary research were carried out on a deeper level, promoting complex and interdisciplinary subjects, such as horizontal disciplines ( informatics ) and Integrated disciplines ( environmental science).From the above discussion, it can be seen that development of disciplines reflects the inherent law of science development to a certain extent. From the history of the disciplinary development, the development of discipline follows the trend of integration-differentiation-integration again. The overall trend of the contemporary discipline is both differentiated and integrated. The system of discipline is not just the static build-up of subjects or discipline, but a dynamic structure of the condensed subject groups, adjusting and combining with the evolution of science.

With the development of disciplines, the forms of academic organizations in colleges and relationships among disciplines are also evolving constantly. The university was basically a 
single college when it was first established. For example, the University of Paris was a theological college and the Italian Bologna University was a college of medical science in the 12 th century. With the development of disciplines, university was established on the basis of the first four disciplines (literature, law, theology, and medicine), such as Oxford University in the 13th century. University was formed on the basis of the traditional four departments, department of liberal arts, department of law, department of theology and department of medicine. Departments didn't provide undergraduate teaching at the time and they were just the academic community of scholars and researchers working on academic activities. From the16th century to the 19th century, a large number of emerging disciplines were created and refined, and the university's academic organizations were changing with it. Established in 1810,Humboldt University of Berlin, in order to meet the new educational goals, created the new "lecture" mechanism, breaking the original academic organizations while retaining the structure of departments at the same time. "Lectures" were seminars at the beginning, and then turned into Institutes, laboratories, and even hospitals. They were in cubators for new subjects and new knowledge. In these new facilities, Humboldt University of Berlin created a mode of education combining academic freedom, research, and teaching. The academic organizational structure of university-department-lecture or institutes was created. In reference to the German university model, universities of the United Kingdom and the United States gradually formed university-college-department structure. Department was established based on a certain discipline. Academic center was focused on the department level. We can see that the 19th century university academic structure in the world was formed as a result of the division of disciplines during this period. From the second half of the 20th century onwards, the development of the discipline showed growth in the research scale of disciplines and cross of research fields. Research methods started to show influence by each other and research fruits started to permeate, which is the start of the interdisciplinary research. In many countries, particularly in developed countries, academic organizations in colleges were being transformed in order to adapt to the development trend of disciplines. A large number of independent interdisciplinary research institutes and research projects were formed. Subjects across disciplines and multi-discipline degrees started to appear.

\section{THE CURRENT STATUS AND ISSUES OF COLLEGE ACADEMIC ORGANIZATIONAL STRUCTURE IN CHINA IN THE PERSPECTIVE OF DISCIPLINES}

\section{A. The Division of Academic Organizations in China Is not Scientific}

Level A discipline is a collection of subjects with relatively common theoretical base or consistent research subject. It is set up according to the subject property. Secondary disciplines are the basic units forming level-A disciplines. Liu shao xue made statistical study of student data at seven universities, including Tsinghua University, Peking University, Fudan University, Nanjing University, Shanghai Jiaotong University, Zhejiang University, Xi' An Jiaotong University. The universities that set up schools according to the discipline category accounted for $31.3 \%$, and those set up schools based on level-A disciplines accounted for $58.8 \%$, while those set up schools based on secondary disciplines accounted for $10 \%$. As a comparison, the top-notch30 universities in the world, such as Princeton University, Yale University, the University of Cambridge, Tokyo University, were set up in the following ratios. Schools that were set up on the basis of level-A discipline accounted for $81 \%$, schools that were set up on the basis of discipline accounted for $3.4 \%$, schools that were set up according to level-A discipline accounted for $11.4 \%$, and schools that were set up according to secondary discipline only accounted for $4.1 \%$. Schools in world-class universities mainly were set up based on the discipline categories, so a school can accommodate many disciplines. Universities in China mainly set up schools based on level-A disciplines, while some local universities set up their schools based on secondary disciplines. It is obvious that universities in China set up schools in a model of professional training. The main task of these schools is teaching. The schools are divided into departments, teaching groups and further refinement, but little consideration is put on the construction of scientific research platforms. Scientific research institutes affiliated with schools are mostly built on the basis of secondary disciplines. The classifications of the academic organizational levels were originated from the adjustment and consolidation of the institutions and schools in the 90 s of the 20th century. The joint universities simply combined several of the original departments to form a school and upgraded the teaching groups to departments, but there was not much change in real terms. The university had two-level or three-level hierarchies. Most of the academic structure retained the traditional department level. The teaching groups, research groups, subject groups, project groups, etc, were re-organized underneath the department structure. This also led to more levels under an academic organizational structure. It is extremely inappropriate for the development of a flat organization. Schools and departments were both organizations sat the intermediate level, which resulted in vague power distribution and work scheduling. The roles of departments and teaching groups were not clear.

\section{B. Academic Organizations in Colleges Lack Flexibility and Dynamism.}

Academic organizations in colleges in China for a long time were not only academic units, but were characterized by a strong executive nature. They were not only responsible for teaching, scientific research, and other academic tasks, but were also responsible for the internal reallocation of resources within the college. The executive powers played a leading role in the academic organizations. The unit-ownership feature dominated by executive power caused lack of effective channels of communication among members of the academic organizations. It was difficult to carry out interdisciplinary research projects. The allocation efficiency of quality research resources was low. It also affected the cohesion of school disciplinary advantages, and it was difficult to highlight the characteristics of colleges. Meanwhile, the executive division was responsible for management functions of academic institutions. The executive division both served the academic institutions as well as carried out administrative functions. This caused academic power and administrative power to be intertwined. The member of academic committee had both 
academic and administrative duties. It was unavoidable that two kinds of power became conflict with each other in many decision-making processes in academic affairs.

The scientific development trend of comprehension-differentiation-integration changes scientific research and innovation activities from individual creation to a collective creation. Members of the organization only have research advantage in a certain field of study. Significant break thoughts in scientific research can only be achieved through cooperation and integration among members. Academic organizations in colleges in China, especially interdisciplinary academic organizations, are lack of long-term mechanism for members to carry out research and development activities for many reasons. Some reasons include that members of the organization belong to different executive offices, or there are limitations in the college recruitment, research evaluation, and remuneration system. This leads to the lack of long-term mechanism to organize research activities within the organization. The division of tasks among members is not clear and there is a lack of close cooperation. Although the organization is formally a research institution, it is still short of coherent power during operation. Phenomena such as lack of exchange of scientific research resources and information, and repeat study on the research projects, often occur.

\section{POLICIES FOR THE CONSTRUCTION OF COLLEGE ACADEMIC ORGANIZATIONS FROM THE PERSPECTIVE OF DISCIPLINES}

\section{A. Establish Academic Organizations in the Form of A Matrix Structure}

As science continues to develop, new disciplines are created. Cross fusion among disciplines have become increasingly prominent. The establishment of the matrix academic organizations can better adapt to the change of disciplines, and continue to improve the science and technology innovation ability in colleges. Matrix academic organization structure is to establish research institutes horizontally across departments, while retaining the original vertical academic organization base, including schools, departments, and teaching groups. This type of academic organization has the main executive administration function in the vertical direction to assure proper completion of education tasks. In the horizontal direction, the research organization across schools, departments, and disciplines are to play the role of academic power. It helps promoting the research fields in different areas and make better integration of the various disciplines that carry out research on major projects. In this organizational structure, a teacher joins the basic academic organization, such as department, teaching groups, etc, based on the type of the course and subject involved in the teaching. At the same time, the teacher can join the basic academic organization that focuses on research, such as discipline groups and research institutes. The different academic organizations that the teacher participates in can exist under the same schools and can also belong to different schools. The academic organization that the teacher joins, especially ones that are oriented in the direction of research duties, can also change dynamically according to the tasks of these academic organizations.

\section{B. The Establishment of Decision-Making Mechanism That}

\section{is Dominated by Academic Power}

University has levels of administrative division, and academic organizations in universities also have the inevitable administrative power in existence. Academic organization has both a hierarchical properties and academic attributes. Hierarchical property emphasizes the establishment of a strict hierarchy and division of powers through executive power. Academic property is based on academic freedom and there is no administrative classification among the members. Academic power plays an important role in the organization operation. Understanding how to solve the contradiction between two different value-oriented functions is also the key to keep academic freedom and independence of the academic organizations. Under the influence of political system and cultural traditions, the university in China is typically dominated by executive power. It relies on an efficient and strict hierarchy based on top-down administrative power to sustain the day-to-day running of the university. In the meantime, the main activities in colleges are academic activities. Academic activities of the base-level organization are characterized by professionalism, independence, and creativity. The decision making should be based on academic management at the core, and the executive administration should be subject to academic needs of academic management. In academic organization, professor committee should be established comprising academic leaders and professors, which should be responsible for the decision making of the academic organizations. College should give professor committee certain extent of power to make academic governance with some room of relative independence. College management with participation of professors can contribute significantly to discipline construction and promote scientific research and innovation in colleges. The establishment of academic-power-driven decision-making mechanism with simultaneous restriction between academic power and administrative power helps create free academic atmosphere and makes complete usage of the basic academic organization's efficiency. Meanwhile, management of the college academic organizations needs to transform its functions constantly and enhances a strong sense of service to provide support and services for the scientific research team. Through reforms in assessment and evaluation mechanism and increase evaluation of the management's services quality, Colleges management can facilitate the upgrade of its service and weaken the abuse of executive power in academic activities.

\section{Improving the Academic Organization's Management System and Create an Organizational Culture that Is Free and Harmonic}

Generally organizational management of university lacks flexibility, and organization operating efficiency is not high. With the mechanism innovation and system reform in colleges, the "one size fits all" management model in human-resources management should be changed. In the management of college headcounts and positions, there should be both fixed positions and rotating positions, and multi-element position-flow patterns combining full-time and part-time staff should be established. At the same time, a healthy entry and exit 
mechanisms should be set up under the uniform standards and constraints of rules. An elastic system of performance assessment should be built to reduce the rigid short-term quantitative evaluation mechanism so that teachers' creativity and vitality can be respected. A more scientific and rational elastic index should be used to guide and motivate the scientific researchers' creative activity. Meanwhile, the evaluation system that is more adapted to the team's development should be explored. An open academic environment that is more adapted to the law of academic research should be established. Any condensed new discipline direction by the team should be protected. On the reform of salary system, various pay systems adapted to the new organizational structure should be explored. A compensation system combining structured salary mechanism and annual wage mechanism, and combing external incentives and the internal incentive, should be established.

Academic organization is an academic community. Members of organizations have a shared vision of academic development and innovation exploration. Learning is the most important attributes of academic organizations. University academic organizations are responsible for important functions such as maintaining the dignity of academics, adhering to strict academic quality, and exploring the academic development. Within the organization, academic freedom should be promoted. Academic exchanges and debates and collision of creative thinking should be encouraged. An efficient organization must have a strong cohesion. Academic organization in colleges and universities should have a unique democratic team-spirit and teams should have close connection to each other and promote each other. Members of the academic organizations develop scientific activities on the basis of common objectives. Members of the academic organizations have close connections, mutual cooperation, mutual respect, and mutual trust to work in harmony. Academic organization members should pass information to each other, understand each other, and create an atmosphere of good communication and coordination within the organization. Free and harmonious culture is an essential condition for academic organizations to make explorations and innovations. In order to create good organizational culture, talent ladder structure can be implemented under the system of academic leaders. Teachers with research subjects belonging to the same discipline category shall be grouped together. The full potential of each teacher's academic expertise can be fully developed by building the core academic discipline and scientific research. Through continuous in-depth research and accumulation, the collective growth of the organization can be achieved eventually.

\section{ACKNOWLEDGMENT}

This article is supported by the Beijing Municipal Education Committee's "RenCaiQiangJiao" Talent Project and "Study on the construction of base-level academic organization at local colleges" project. Project leader is Zhou Min.

\section{REFERENCES}

[1] Tang YuguangPan Qi. The disciplinelogic of university's academic of organizational transformation [J]. Study on the development of education, 2010(19):8-11
[2] Liu shaoxue, Cheng Ying, Dong yuchang, Liu Niancai Innovative layout of discipline, specification in the construction of schools and departments $[\mathrm{J}]$. Study on the education, Tsinghua University,2003(10):70-74

[3] Xiang dongchun, perspective of academic organizationalproperties base level of the University[J]. Higher Engineering Education and Research, 2006(3):104-106 